\title{
Nível de Atividade Física e Hábitos Alimentares de Alunos do Ensino Médio em uma Escola Pública
}

\author{
Physical activity level and eating habits of high school students in a public \\ school
}

\author{
Giuliano Roberto da Silva ${ }^{1}$, Edilson Tadeu Ferreira Furtado², Talece Nascimento ${ }^{3}$, \\ Thaisa Silva Nascimento ${ }^{3}$
}

${ }^{1}$ Faculdade Presbiteriana Gammon- FAGAMMON, Lavras, Minas Gerais. Universidade José do Rosário Vellano- UNIFENAS, Alfenas, Minas Gerais. Centro Universitário do Sul de Minas Gerais- UNISMG, Varginha, Minas Gerais. Universidade Faculdades Metropolitanas Unidas- UNIFMU, São Paulo. Universidade Federal de Lavras- UFLA, Lavras, Minas Gerais. Universidade Vale do Rio VerdeUNINCOR, Minas Gerais. Universidade de Franca- UNIFRAN, São Paulo.

${ }^{2}$ Faculdade Presbiteriana Gammon- FAGAMMON, Lavras , Minas Gerais. Universidade Gama FilhoUGF, Rio de Janeiro. Universidade Federal de Lavras - UFLA, Lavras, Minas Gerais.

${ }^{3}$ Faculdade Presbiteriana Gammon- FAGAMMON, Lavras, Minas Gerais.

\begin{abstract}
Resumo
Introdução: A adolescência é uma fase que vem acompanhada por vários fatores psicológicos, biológicos além da influência por parte da mídia e estudos realizados apontam um aumento na obesidade, advindo da má alimentação e da ausência da prática de atividade física, principalmente, em alunos do ensino médio. Objetivo: Logo, o objetivo do estudo foi verificar a incidência da prática de atividade física de alunos do ensino médio, seus hábitos alimentares, e também avaliar o nível de atividade física e o consumo de alimentos in natura e alimentos industrializados por estes alunos por meio de um questionário qualitativo. Metodologia: A amostra da pesquisa foi composta por 49 alunos do Ensino Médio, de uma escola pública, com idade entre 15 a 20 anos. Para a coleta de dados foram utilizados dois questionários, sendo eles, IPAC versão curta, para avaliar o nível de atividade física, e o Questionário de Frequência Alimentar (QFA) para saber com que frequência são ingeridos alimentos in natura e alimentos industrializados por esses alunos. Resultados: Ao correlacionar os resultados foi possível avaliar que a ingestão de alimentos seja ele industrializado ou in natura é inversamente proporcional aos níveis de atividade física, e não foi possível perceber estatisticamente uma maior incidência de prática de atividade física de um gênero sobre outro. Conclusão: Conclui-se que o consumo de alimentos in natura são pouco ingeridos por essa amostra, havendo maior ingestão de alimentos industrializados.
\end{abstract}

Palavras - chave: Atividade física. Alimentação. Alunos.

Autor correspondente:

Giuliano Roberto da Silva

E-mail: giumusc@gmail.com
Recebido em: 14/05/2016

Revisado em: 17/05/2016

Aceito em: 24/05/2016

Publicado em: 15/06/2016 


\begin{abstract}
Introduction: Adolescence is a stage that is accompanied by various psychological factors, biological beyond the influence by the media and studies show an increase in obesity, resulting from poor diet and lack of physical activity, especially among high school students. Objectives: Therefore, the aim of the study was to determine the incidence of physical activity of high school students, their eating habits, and also assess the level of physical activity and consumption of fresh food and processed food for these students through a qualitative questionnaire. Methodology: The survey sample consisted of 49 high school students in a public school, aged 15 to 20 years. For data collection were used two questionnaires, namely, IPAC short version, to assess the level of physical activity, and the Food Frequency Questionnaire (FFQ) to know how often are eaten fresh food and processed food for these students. Results: By correlating the results was possible to evaluate the food intake whether industrialized or in kind is inversely proportional to the levels of physical activity, and was not statistically realize a higher incidence of physical activity of one gender over another. Conclusion: It is concluded that the consumption of fresh food are eaten by little this sample, with higher intake of processed foods.
\end{abstract}

Keywords: Physical Activity. Alimentation. Students.

\section{Introdução}

De acordo com Domingues ${ }^{1}$ e Knuth et $\mathrm{al}^{2}$, a sociedade em geral tem se conscientizado que praticantes de atividade física têm ganhos em relação à qualidade de vida, na prevenção de doenças crônicas e no seu tratamento. Apesar das pessoas saberem dos benefícios da prática física, o número de não praticantes, ainda, é bem extenso ${ }^{3}$.

Segundo a recomendação da Organização Mundial de Saúde (OMS), o nível adequado de atividade física é de 150 minutos por semana, sendo esta de intensidade leve a moderada ou, então, 75 minutos de intensidade alta ${ }^{4}$.

Para De Angelis ${ }^{5}$, o Brasil é um dos países em que o número de obesos vem aumentando desordenadamente e este aumento associa-se ao estilo de vida da população, em relação à atividade física e sua alimentação. Para tentar mudar essa situação, a adoção de um estilo de vida mais ativa fisicamente deverá ser iniciada ainda nos primeiros anos de vida, com as crianças e adolescentes para que estes jovens tomem consciência da importância de um estilo de vida saudável, e, que mantenham isso no decorrer de sua vida ${ }^{6}$. É sabido que a prática regular da atividade física, juntamente a uma alimentação adequada, são fatores que, quando integrados, podem proporcionar um estilo de vida mais saudável ${ }^{7}$.

A ingestão inadequada de nutrientes, seja por excesso ou insuficiência, aumenta o risco das Doenças Crônicas Não Transmissíveis (DCNT) em se manifestarem ao longo da vida, embora essas não sejam transmissíveis, mortes são frequentes, mediante a isto, a ingestão de alimentos saudáveis é de fundamental importância para prevenir ou controlar várias doenças ${ }^{8}$.

Segundo Mendonça ${ }^{9}$, uma alimentação adequada deve ser estabelecida desde o aleitamento materno, pois é, nessa etapa da vida, que é educado e determinado bons hábitos alimentares nos filhos pela sua Mãe.

Em relação à alimentação dos adolescentes ${ }^{10}$, relata que em busca do corpo perfeito, estes se alimentam de forma desequilibrada, aumentando o risco de transtornos alimentares. E ainda descrevem que no sexo feminino, isso é ainda pior podendo chegar a 90\%, na busca de um emagrecimento extremo $^{10}$.

Os autores Fisberg et at ${ }^{11}$, ao observarem as preferências alimentícias dos jovens, comprovaram que os alimentos inadequados e com carências nutricionais estão cada vez mais presentes na alimentação cotidiana desta faixa etária. Devido a isso o estudo de Oliveira et $\mathrm{al}^{12}$, demonstrou que há um aumento de doenças relacionadas a uma alimentação inadequada entre os jovens.

A Agência Nacional de Vigilância Sanitária ANVISA $^{13}$, descreve que é de suma importância que os indivíduos mudem seus hábitos alimentares progressivamente, e, ainda, modifiquem a composição nutricional de cada alimento. Dessa forma, alimentos como açúcares, gorduras saturadas, gordura trans e sódio seriam menos inseridos na alimentação diária e contribuiria para a melhora da qualidade de vida. 
Em 2006, o Programa Nacional de Alimentação Escolar (PNAE) ${ }^{14}$, em conjunto com as escolas públicas do Brasil, estabeleceram estratégias de hábitos saudáveis na alimentação dos alunos. Mediante a isso, o objetivo deste estudo foi verificar a incidência da prática de atividade física de alunos do ensino médio, e sua relação com os hábitos alimentares, e também avaliar o nível de atividade física e o consumo de alimentos in natura e alimentos industrializados por estes alunos por meio de um questionário qualitativo em uma escola pública.

\section{Metodologia}

O estudo realizou-se em uma escola pública estadual da cidade de Lavras - MG. Essa escola funciona no período diurno (séries I, II, III do Ensino Médio e os anos oitavo e nono do Ensino Fundamental II), vespertino (Ensino Fundamental I e II), e noturno (Educação Especial de Jovens e Adultos - EJA). A pesquisa foi ministrada no período diurno, com alunos do ensino médio. Foram avaliados 49 estudantes sendo 29 do sexo feminino e 20 do sexo masculino, com idade entre 15 a 20 anos.

Para realização da pesquisa foram utilizados dois termos de aceitação, sendo eles o Termo de Consentimento Livre e Esclarecido (TCLE) para que os alunos obtivessem autorização de seus pais para participarem da pesquisa e o Termo de Assentimento Informado Livre e Esclarecido (TAILE) para os próprios alunos estarem cientes de sua participação na pesquisa.

Foi realizada uma pesquisa de campo de caráter quantitativo e descritivo e foram utilizados dois questionários: um para avaliar o nível de atividade física destes alunos, sendo ele o IPAC versão curta, já que este questionário e de fácil aplicação, baixo custo e recomendado pela literatura para medir o grau de sedentarismo da população, seja dentro ou fora do ambiente escolar; e o outro para avaliar qualitativamente o consumo alimentar, sendo este o Questionário Frequência Alimentar (QFA).

O critério de inclusão para participação no estudo foi os alunos estarem matriculados e frequentes no Ensino Médio, e os critérios de exclusão foram: alunos que não obtiveram autorização de seus pais e alunos que não estivessem em aula no dia da coleta de dados.

Este estudo ofereceu mínimos riscos aos participantes, já que não se trata de medidas invasivas ou testes de esforço físico. Um provável risco seria de algum aluno se sentir incomodado durante as respostas dos questionários.

Ao final da pesquisa, os dados foram analisados por meio do software Microsoft Excel 2010 e demonstrados através de gráficos, utilizando a estatística descritiva simples. Para obtenção dos coeficientes de correlação dos resultados, foi utilizada a função Correl do Microsoft Excel 2010, e para comparação dos resultados, foi utilizado o teste $\mathrm{t}$ de Student, considerando $\mathrm{p} \leq 0,05$.

\section{Resultados}

Após análise dos dados coletados, apurou-se em relação ao consumo de alimentos in natura (frutas), que apenas $16,3 \%$ da amostra consomem de duas ou mais porções de frutas por dia, que é o recomendado. Já $83,7 \%$ da amostra não fazem o consumo de porções de frutas recomendadas por dia (Figura 1).

Figura 1 - Consumo de alimentos in natura (frutas).

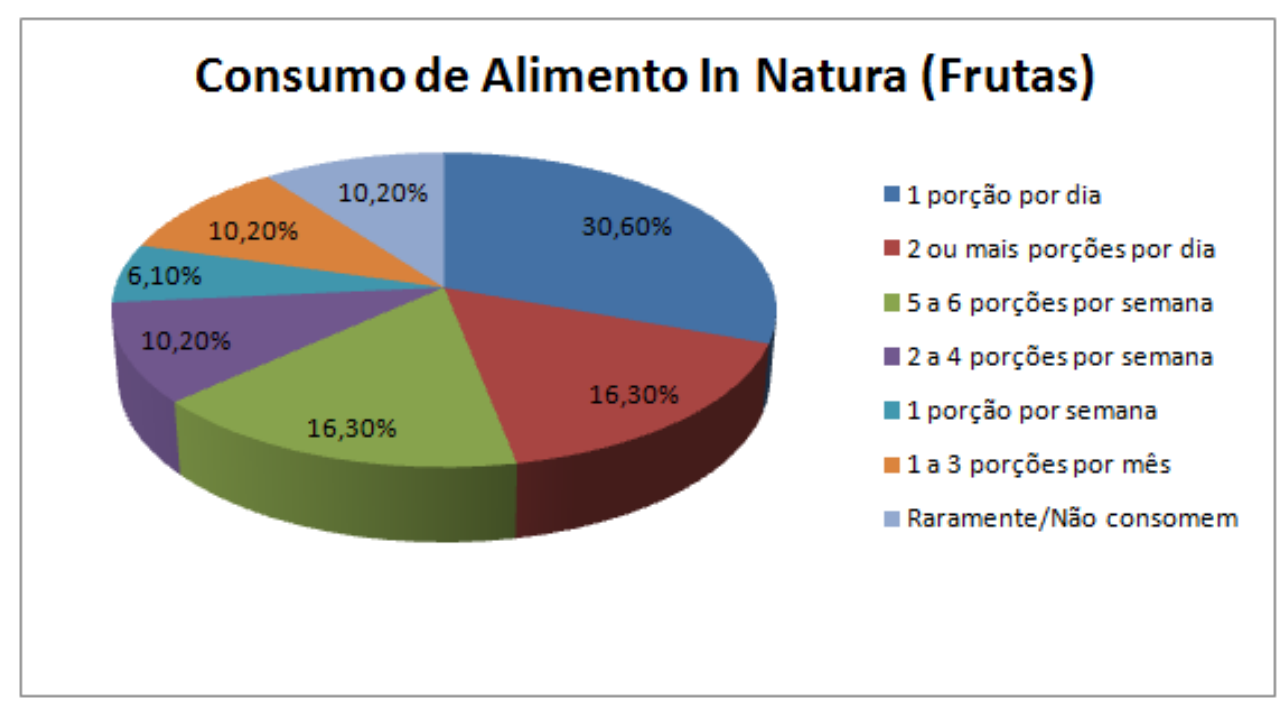

No que diz respeito ao consumo, em maior quantidade, de alimentos industrializados (snacks) pelos adolescentes estudados, $14,3 \%$ consomem uma vez ao dia, enquanto $2,0 \%$ consomem duas ou mais vezes ao dia, e $6,1 \%$ consomem de cinco a seis vezes por semana. Porém, nota-se que $26,5 \%$ da amostra, não consomem este tipo de alimento (Figura 2). 
Figura 2 - Consumo de alimentos industrializados (snacks).

\section{Consumo de alimentos industrializados (snacks)}

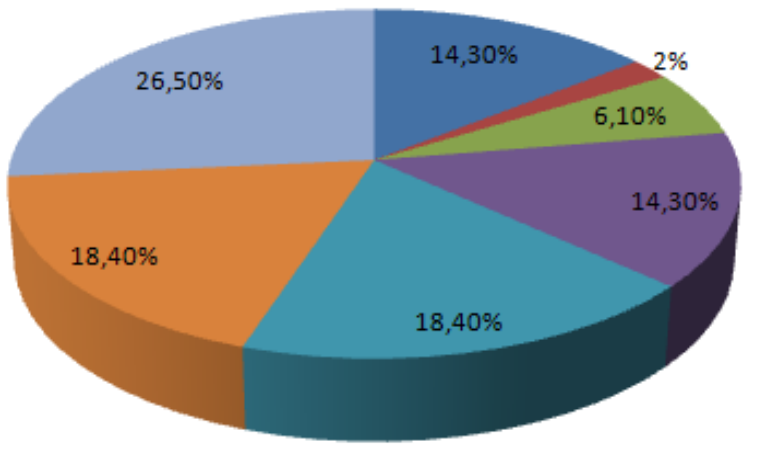

1 vez ao dia

- 2 ou mais vezes ao dia

5 a 6 vezes por semana

2 a 4 vezes por semana

1 vez por semana

1 a 3 vezes por mês

naramente/Não consomem

No que tange o consumo de alimentos industrializados (sanduíches), os resultados mostraram que $14,3 \%$ dos alunos consomem sanduíches uma vez ao dia, enquanto que 2,0\% dos alunos consomem sanduíches duas ou mais vezes por dia. Logo, pode-se perceber que o consumo deste tipo de alimento não é tão frequente entre a amostra estudada (Figura 3).

Figura 3 - Consumo de alimentos industrializados (sanduíches).

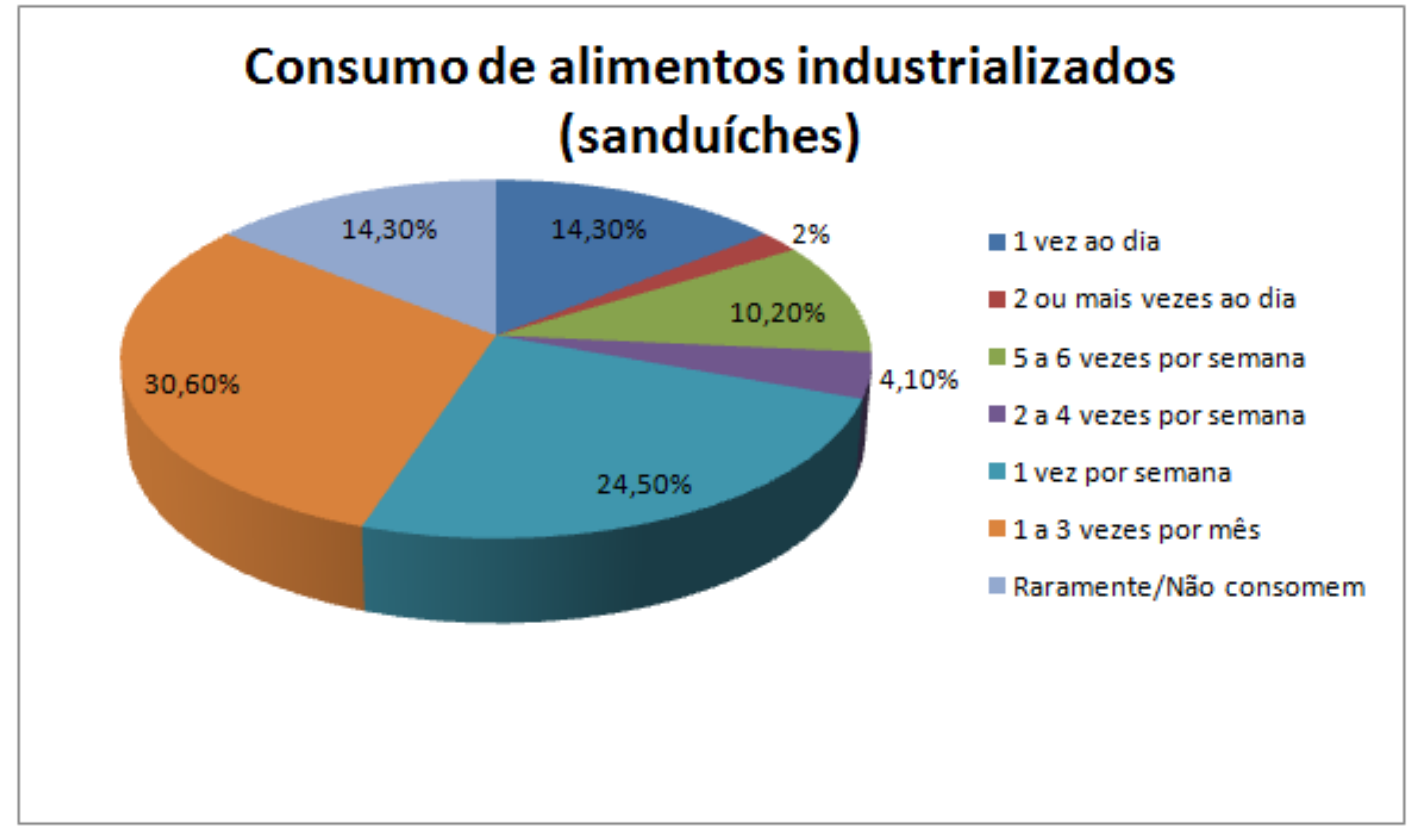

Na Figura 4, pode-se observar em relação ao consumo de refrigerantes, que $10,2 \%$ dos alunos consomem uma, duas ou mais vezes ao dia refrigerantes. Mostrando que o consumo desta bebida não é tão usual entre os hábitos dos estudados. 
Figura 4 - Consumo de alimentos industrializados (refrigerantes).

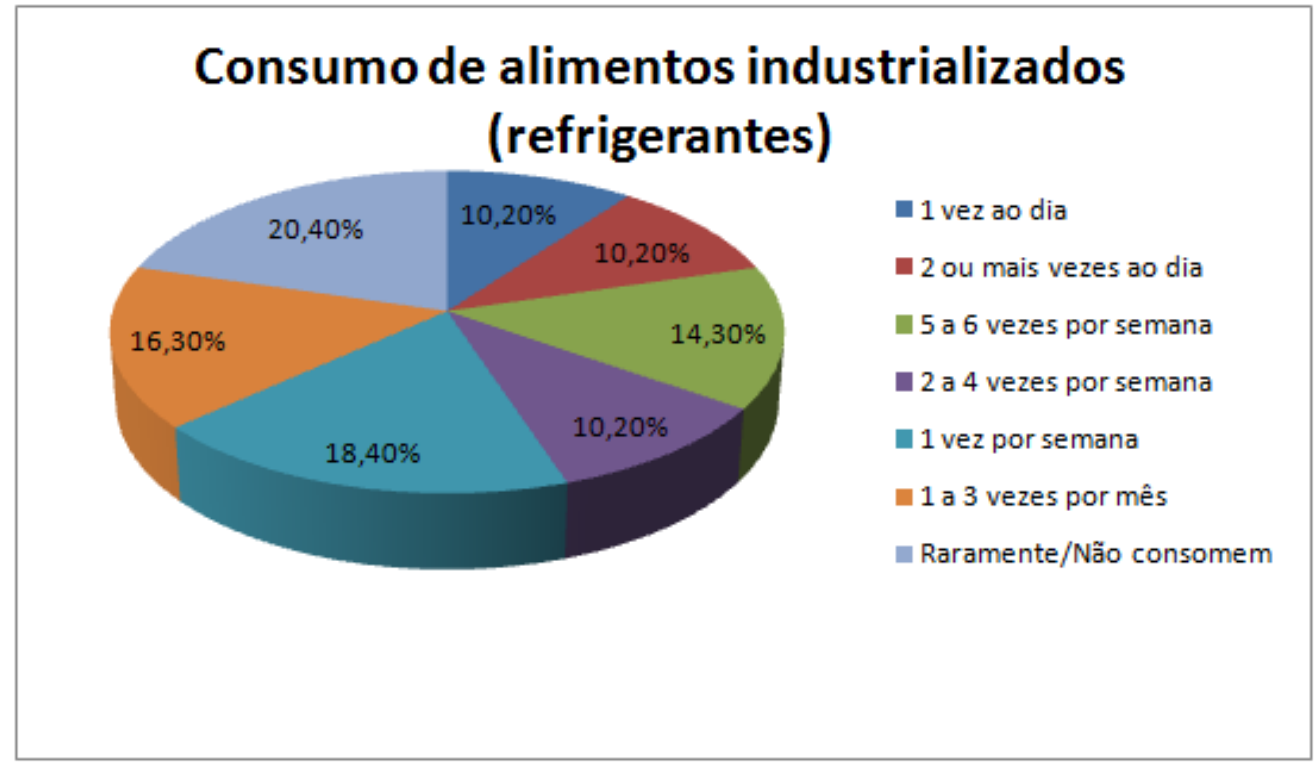

Ao serem questionados sobre seus níveis de atividade física, 69,4 \% dos alunos são considerados altamente ativos; 24,5 \% considerados suficientes ativos, enquanto que $6,1 \%$ são considerados insuficientes ativos (Figura 5).

Figura 5 - Nível de atividade física.

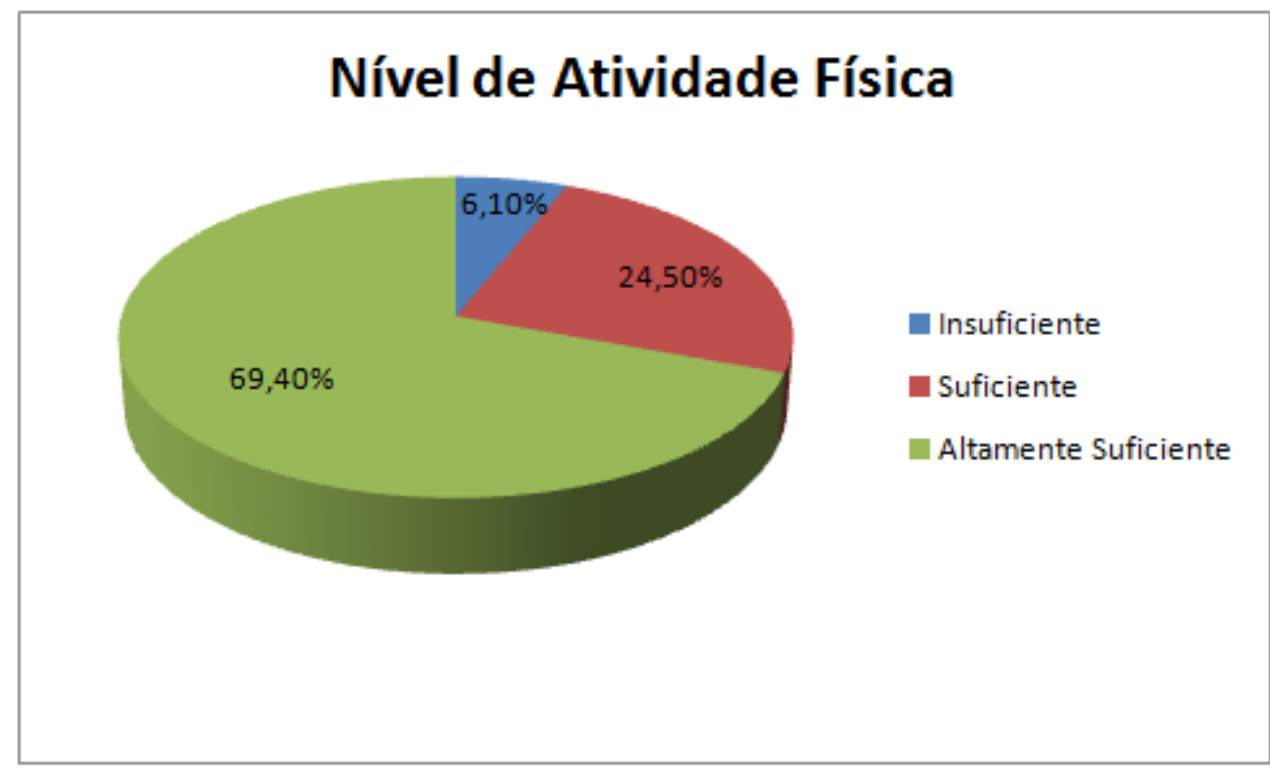

Ao correlacionar estatisticamente à ingestão de alimentos tanto industrializados quanto in natura (frutas) com os níveis de atividade física dos participantes da pesquisa, foi possível constatar uma correlação muito fraca (CMF), ou seja, as duas variáveis são inversamente proporcionais, onde quem consome mais ou menos alimentos industrializados não correlaciona com quem possui o nível de atividade física insuficiente ou altamente insuficiente, respectivamente, e quem consome mais ou menos alimentos in natura não correlaciona com quem possui o nível de atividade física altamente suficiente ou insuficiente, respectivamente (Tabela 1). 
Tabela 1 - Correlação entre as variáveis alimentares versus atividade física.

\begin{tabular}{cl}
\hline Frutas x Atividade Física & $\mathbf{- 0 , 9 3 4 0 9}(\mathrm{CMF})$ \\
\hline Snackers x Atividade Física & $\mathbf{0 , 0 0 4 7 5 8}(\mathrm{CMF})$ \\
Sanduíches x Atividade Física & $\mathbf{- 0 , 2 6 5 9 2 0 9}(\mathrm{CMF})$ \\
Refrigerantes x Atividade Física & $\mathbf{- 0 , 2 3 4 9 3 7 5 7}(\mathrm{CMF})$ \\
\hline
\end{tabular}

Ao comparar os níveis de atividade física entre os gêneros estudados, notou-se que 2,0 \% dos meninos são consideradas insuficientes ativos; 10,2 $\%$ dos meninos são considerados suficientes ativos; $28,6 \%$ dos meninos são considerados altamente ativos. Já em relação às meninas, $4,1 \%$ são consideradas insuficientes ativas; $14,3 \%$ das meninas são consideradas suficientes ativas; $40,8 \%$ das meninas são consideradas altamente ativas (Figura 6). Porém, os gêneros ao serem comparados estatisticamente, não houve diferença significativa ( $\mathrm{p}$ $=0,672$ ), logo, não se pode relatar que um gênero possui nível de atividade física maior que o outro.

Figura 6 - Nível de atividade física entre os gêneros.

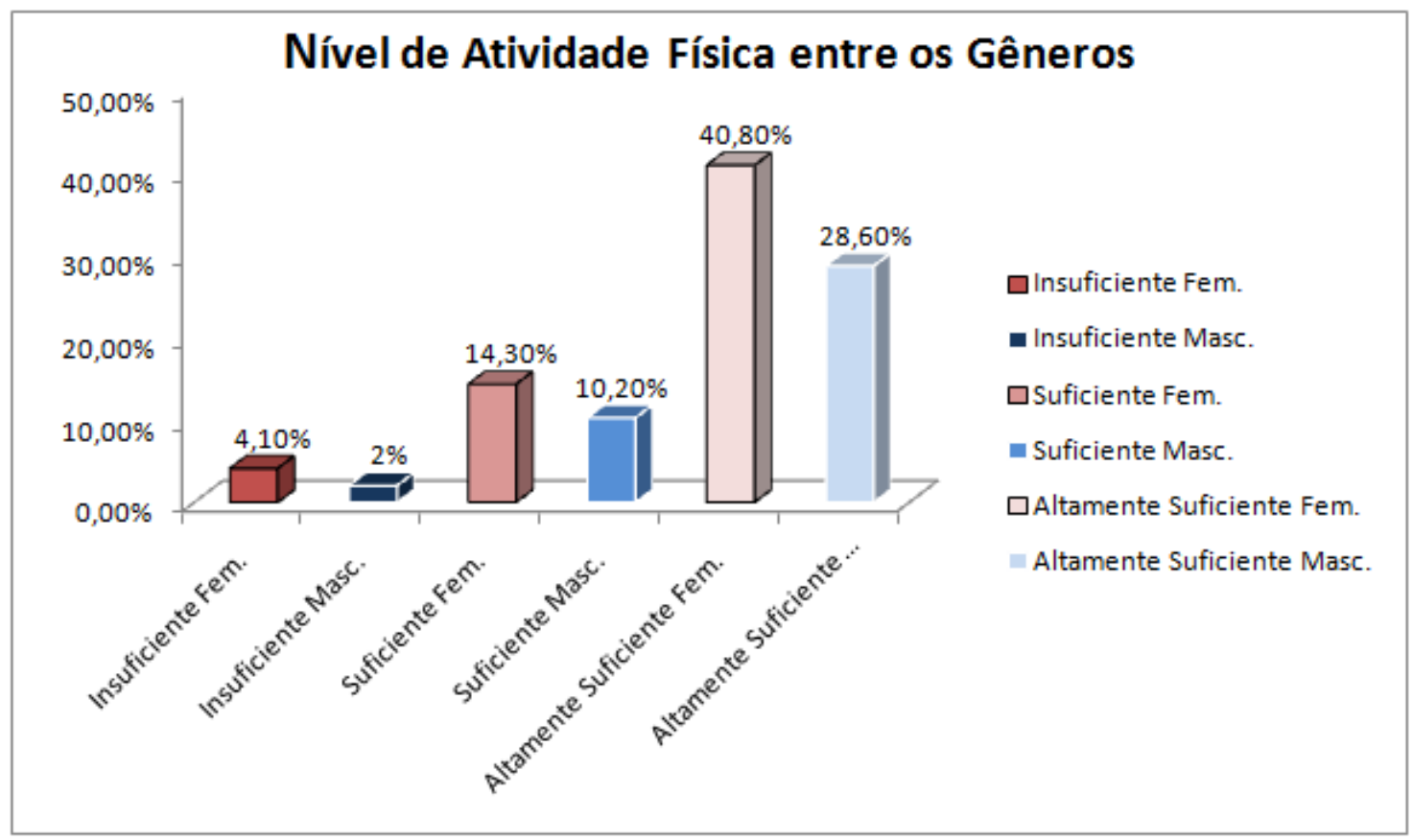

\section{Discussão}

A pirâmide alimentar (Figura 7), criada pela Organização Mundial de Saúde, e adaptada a população brasileira tem como objetivo incentivar indivíduos a se alimentarem de forma saudável, e no mesmo instante, ajudar para a escolha correta dos alimentos e o seu consumo em porções diárias. Esta se divide em quatro níveis, e estes, em oito grupos. Começando da base para o ápice da pirâmide estão os carboidratos que compõem o primeiro nível; no segundo nível, e também segundo grupo, estão as hortaliças e vegetais. As frutas se encontram no terceiro grupo e segundo nível sendo aconselhável um consumo entre 3-5 porções ao dia; já o leite, iogurtes e queijos, estão no terceiro nível e quarto grupo; no quinto grupo estão às carnes, aves, peixes, ovos; o feijão, nozes se localizam no terceiro nível e sexto grupo. As gorduras e os óleos, assim como os doces e açúcares estão no sétimo e oitavo grupo respectivamente. $\mathrm{O}$ consumo dos alimentos contidos no último nível desta pirâmide deve ser limitado e restrito a poucas quantidades ao dia. 
Figura 7 - Pirâmide alimentar.

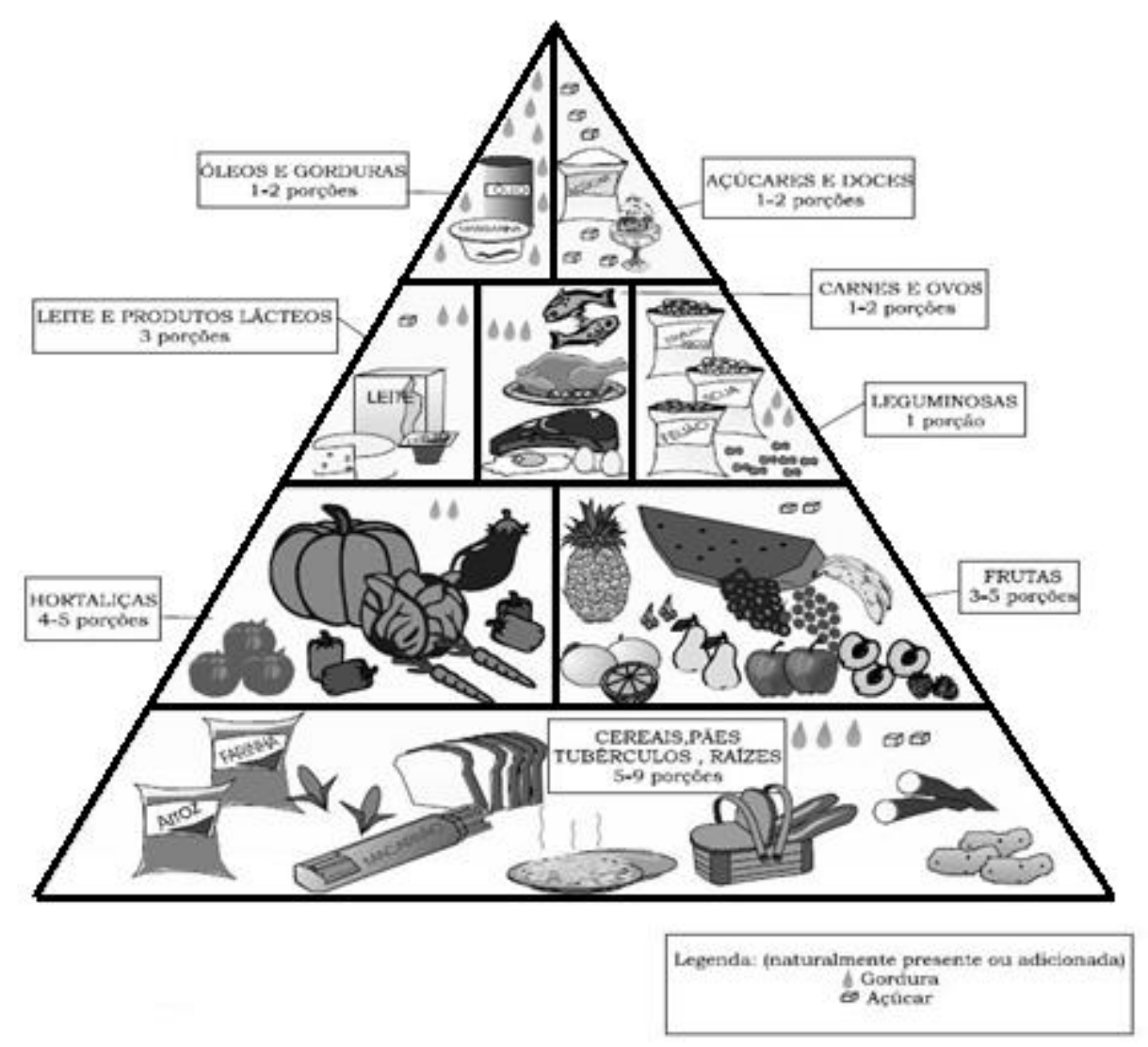

Fonte: PHILIPPI, 2008.

Os resultados apresentaram que $83,6 \%$ dos alunos não consomem a quantidade de fruta que são recomendados por dia, dados esses preocupantes. $\mathrm{O}$ consumo diário de frutas deve ser de três porções diárias, já que as frutas são fontes de vitaminas, fibras e minerais, que proporcionam o bom funcionamento do nosso organismo prevenindo doenças e auxiliando no combate das mesmas. Isto se dá pelo seu baixo teor calórico e de gordura, e por serem ricas em fibras, contribuindo assim para um menor consumo de outros alimentos, dado a sensação de saciedade que as fibras proporcionam ${ }^{15}$.

Em relação à ingestão de alimentos industrializados: snacks e sanduíches, em que 14,3\% dos alunos consomem esses alimentos uma vez ao dia, e $2 \%$ dos alunos consomem esses alimentos duas ou mais vezes ao dia. Os lipídeos assim como outros alimentos, possuem funções importantes no organismo, de reserva energética, constituição de membranas celulares e absorção de algumas vitaminas, no entanto, são insubstituíveis e indispensáveis ao bom funcionamento do organismo; por outro lado, o consumo excessivo de alimentos ricos em lipídeos está associado às $\mathrm{DCNT}^{16}$. O consumo excessivo destes alimentos em longo prazo pode trazer prejuízos irreversíveis a esses adolescentes $^{17}$.

Dos adolescentes estudados, 10,2\% consomem refrigerantes pelo menos uma vez ao dia, também $10,2 \%$ destes chegam a ingerir duas ou mais vezes por dia. Por este alimento possuir um alto índice de carboidrato simples (açúcar), não deveria ser ingerido com tanta frequência, já que ele tem ligação direta com a obesidade e a diabetes ${ }^{18}$.

Sobre os benefícios proporcionados pele atividade física, estes são diversos, e vão desde a prevenção de doenças até o seu tratamento. De acordo com o Questionário aplicado IPAC, classificam-se como pessoas "insuficientes ativas" indivíduos que fazem até 600 METS por semana, indivíduos "suficientes ativos" que praticam de 600 METS a 1500 METS semanais, e "altamente ativos" aqueles indivíduos que praticam acima de 1500 METS semanais.

Notou-se que 6,1\% (n=3) da amostra estudada são considerados insuficiente ativos. Em um estudo de Junior et al $^{19}$, apontam que, quando indivíduos são praticantes de atividade física na adolescência, a 
probabilidade de se tornarem adultos ativos são maiores. Portanto, o incentivo à prática de atividade física, nessa fase da adolescência, torna-se um fator importante para reduzir os níveis de sedentarismo na vida adulta.

O presente estudo demonstrou que 93,8\% $(n=46)$ dos adolescentes entrevistados foram considerados ativos ou altamente ativos, dados esses que se assemelham ao estudo de Ribeiro et $\mathrm{al}^{20}$, na cidade de Pelotas/RS. Uma hipótese pelo resultado apresentado se dá pela participação da escola em projetos esportivos como, atletismo, basquete, voleibol, futsal.

O estudo apresentou, que as meninas estão mais ativas em relação aos meninos tanto na classificação "suficiente ativo" como também "altamente ativo", o que difere do estudo de Guedes et $\mathrm{al}^{21}$ e Santos et $\mathrm{al}^{22}$, onde apresentaram que os meninos são mais ativos em relação às meninas. $\mathrm{O}$ resultado do presente estudo se deu devido a maior participação das meninas nos projetos esportivos da escola pesquisada.

\section{Conclusão}

De acordo com os resultados encontrados, a maioria dos gêneros está classificada como fisicamente ativos, porém ao serem comparados estatisticamente, um gênero não se sobressaiu sobre outro.

Dos alimentos avaliados, notou-se uma grande incidência por alimentos industrializados, que são ricos em carboidratos simples (açúcares) e gordura, tanto saturada quanto trans. Já no que se refere ao consumo de frutas, este ficou a desejar, havendo um consumo inadequado de acordo com a recomendação.

Através da correlação, foi possível avaliar que a ingestão de alimentos seja ele industrializado ou in natura é inversamente proporcional aos níveis de atividade física, ou seja, quem pratica mais atividade física não necessariamente consume menos alimentos industrializados e mais alimentos in natura.

Assim, como na atividade física, seria interessante que os alunos melhorassem seus hábitos alimentares para terem um estilo de vida saudável, de modo a atuarem na prevenção das doenças crônicas não transmissíveis.

\section{Declaração de conflitos de interesses}

Os autores declaram não haver conflito de interesses.

\section{Referências}

1. DOMINGUES, M. R. Conhecimento populacional sobre atividade física. In: Florindo AA, Halla IPC (eds).
Epidemiologia da Atividade Física. São Paulo: Editora Atheneu; 2011. p.139-150.

2. KNUTH, G. A.; BIELEMANN, M. R.; SILVA, G. S.; BORGES, T. T.; DEL DUCA, G. F.; KREMER, M. M. Conhecimento de adultos sobre o papel da atividade física na prevenção e tratamento do diabetes e hipertensão. Estudo de base populacional no sul do Brasil. Caderno de Saúde Pública. v.25, p. 513-20, 2009.

3. BARRETO, M. S.; PINHEIRO, A. R. O.; SICHIRI, R.; MONTEIRO, C. A.; BATIST FILHO, M.; SCHIMITD, M. I. Análise da Estratégia Global para Alimentação, Atividade física e Saúde, da Organização Mundial da Saúde. Epidemiol Serv Saúde. v.14, p. 41- 68, 2005.

4. ORGANIZAÇÃO MUNDIAL DA SAÚDE (OMS). Prevenção de doenças crônicas: um investimento vital. Brasília: Organização Pan-Americana da Saúde. 2013.

5. DE ANGELIS, R. C. Obesidade: prevenção nutricional. Nutrição em Pauta. 2005. v. 72: 4-8.

6. NEUMARK-SZ TAINER, D.; STORY, M.; HANNAN, P. J.; THARP, T.; REX, J. Factors associated with changes in physical activity: a cohort study of inactive adolescent girls. Archives of Pediatrics and Adolescent Medicine. v.157: 803-810. DOI: 10.1001/archpedi.157.8.803, 2003.

7. MARCONDELLI, P.; SCHMITZB, A. S.; COSTA, T. H. M. Nível de atividade física e hábitos alimentares de universitários do $3^{\circ}$ e $5^{\circ}$ semestres da área de saúde. Revista nutrição. v.21, p.39-47, 2008.

8. VIANA, V.; SANTOS, P. L.; GUIMARÃES, M. J. Comportamento e hábitos alimentares em crianças e jovens. Uma revisão da literatura. Psic., Saúde e Doenças. v.9, n.2, p.168-177, 2008.

9. MENDONÇA, R. T. Nutrição: um guia completo de alimentação, práticas de higiene, cardápios, dietas, gestão. 1 ed. São Paulo: Rideel, 2010.

10. RODRIGUES, A. M.; FISBERG, M.; CINTRA, I. P. Avaliação do estado nutricional, prevalência de sintomas de anorexia nervosa e bulimia nervosa e percepção corporal de modelos adolescentes brasileiras. Nutrição Brasil. v.4, n.4, p.182 -187, 2005.

11. FISBERG, M.; BANDEIRA, C. R. S.; BONILHA, E. A.; HALPERN, G.; HIRCHBRUCH, M. D. Hábitos alimentares na adolescência. Pediatria Moderna. v. 36, p. 724-34, 2000.

12. OLIVEIRA, C. L.; MELLO, M. T.; CINTRA, I. P.; FISBERG, M. Obesidade e síndrome metabólica na infância e adolescência. Revista de Nutrição. v. 17, n. 2, p. 237-245, 2004.

13. AGÊNCIA NACIONAL DE VIGILÂNCIA SANITÁRIA (ANVISA). Disponível em: www.anvisa.gov.br. Acesso em: 24 de Abril de 2015.

14. PROGRAMA DE ALIMENTAÇÃO ESCOLAR (PNAE). 2006. Disponível em: http://www.fnde.gov.br/home/. Acesso em: 26 de Maio de 2015. 
15. NUTRITOTAL. Coma mais frutas. 2015. Disponível em: www.nutritotal.com.br. Acesso em: 19 de Outubro de 2015.

16. FIORINI, P. S.; LORENCINI, A. J. Atividades de aprendizagem sobre nutrição: implicações para a reeducação alimentar dos alunos. O professor PDE e os desafios da escola pública paranaense. Governo do Estado - Secretaria da Educação, Paraná. v.1, p. 69-77, 2012.

17. SALES, F. H. S.; CARVALHO, W. R. C.; JUNIOR, J. M. S.; SILVA, D.C.; SANTOS, C. M. Maus hábitos alimentares de estudantes do ensino médio em escolas públicas. HOLOS. v. 30, n. 4, p.247-255, 2014.

18. ROSADO, E. L.; MONTEIRO, J. B. R. Obesidade e a substituição de macronutrientes da dieta. Rev. Nutr. v.14, n.2, p.145-152, 2001.

19. JUNIOR, M. R. A.; ARAUJO, C. L. P.; PEREIRA, F. M. Atividades físicas e esportivas na adolescência: mudanças de preferências ao longo das últimas décadas. Rev. Bras. Educ.fis. v.20, n.1, p.51-58, 2006.

20. RIBEIRO, J. A. B.; CAVALLI, A. S.; CAVALLI, M. O.; AFONSO, M. R. Nível e importância atribuídos a prática de atividade física por estudantes do ensino fundamental de uma escola pública de Pelotas/RS. Revista Mackencie de Educação Física e Esporte. v.12, n.2, p. 13-25, 2013.

21. GUEDES, D. P.; GUEDES, J. E. R. P.; BARBOSA, D. S.; OLIVEIRA, J. A. Níveis de prática de atividade física habitual em adolescentes. Revista Brasileira de Medicina do Esporte. v.7, n.6, p. 123-134, 2001.

22. SANTOS, S. M.; HINO, F. A. A.; REIS, S. R; ANEZ, R. R. C. Prevalência de barreiras para a prática de atividade física em adolescentes. Rev Bras Epidemiol. v.13, n.1, p. 94-104, 2010. 